\title{
A IMPORTÂNCIA DO PROFISSIONAL DA ENFERMAGEM NA PREVENÇÃO DO CÂNCER DO COLO DE ÚTERO NA SAÚDE DA MULHER: uma revisão de literatura
}

Gleice Fernandes de SOUSA $^{1}$

Débora de Fátima Mendonça Santos CAVALCANTI²

\begin{abstract}
${ }^{1}$ Bacharel em Enfermagem - Centro Universitário UNINOVAFI; Pós Graduanda em Saúde da Família - Centro Universitário Estácio de Sá. E-mail: gleicygue@ hotmail.com

${ }^{2}$ Mestre em Saúde da Família pelo Centro Universitário UNINOVAFAPI; pós-graduação em Osteopatia pela Faculdade de Ciências Médicas de Minas Gerais; pós-graduação em Traumato-ortopedia e Desportiva pela Faculdade de Tecnologia Internacional (2009), e Formação Completa em Osteopatia Estrutural pela Escola Brasileira de OsteopatiaEBOM. Graduada em Fisioterapia pela Faculdade Integral Diferencial - FACID. Docente da FATESP e FAMEP. Coordenadora geral dos cursos técnicos da CETESP. Membro do Grupo de Estudos em Saúde da Família GESF do UNINOVAFAPI. E-mail: debyfms@ hotmail.com
\end{abstract}

Recebido em: 14/01/2016 - Aprovado em: 0511/2016 - Disponibilizado em: 18/12/2016

\begin{abstract}
RESUMO:
O câncer cérvico-uterino tornou-se alvo da comunidade científica por apresentar elevadas taxas de morbidade e mortalidade entre a população feminina, especialmente nos países em desenvolvimento, nos quais esse tipo de câncer relaciona-se ao perfil epidemiológico das mulheres. A pesquisa teve como objetivos avaliar os artigos publicados na área da enfermagem e revista especializadas em saúde da mulher, demonstrar como a enfermagem é importante na atenção básica com relação à prevenção do câncer de colo de útero na mulher; identificar as pesquisas relacionadas à prevenção do câncer do colo de útero; observar quais as contribuições apresentadas nas pesquisas em estudo e demonstrar a importância da detecção precoce na atenção básica na maioria das vezes feita pelo profissional da enfermagem. Trata-se de um Trata-se de uma pesquisa bibliográfica de cunho qualitativo onde foi utilizado como base de dados online Scielo, livros e sites do Ministério da Saúde. Os resultados mostraram que a maioria dos casos de detecção do câncer do colo do útero foi feito por enfermeiros em UPA'S, mostrando assim como o conhecimento cientifico a cerca do câncer de colo do útero por parte deste profissional é relevante no que se refere ao manejo, tratamento, rastreamento e promoção de saúde da mulher.
\end{abstract}

Palavras-chave: câncer do colo do útero. Cuidado. Enfermagem.

\begin{abstract}
:
The cervical cancer has become the target of the scientific community by presenting high rates of morbidity and mortality among women, especially in developing countries, where this type of cancer is related to the epidemiological profile of women. The research aimed to evaluate the articles published in the field of nursing and specialized magazine on women's health, demonstrate how nursing is important in primary care regarding prevention of cervical cancer in women; identify research related to the prevention of cervical cancer; observe that the contributions made in the research study and demonstrate the importance of early detection in primary care for the most part made by professional nursing. This is a This is a bibliographic research of qualitative approach which was used as online database Scielo, books and websites of the Ministry of Health. The results showed that most cases of detection of cervical cancer was made by nurses in UPA'S, showing as well as scientific knowledge about cervical cancer by this professional is relevant with regard to the handling, treatment, screening and promotion of women's health.
\end{abstract}

Keywords: Cervical cancer. Care. Nursing. 


\section{CONSIDERAÇÕES INICIAIS}

Este artigo cientifico se insere na temática da relevância do profissional da enfermagem na prevenção do câncer cérvico-uterino na unidade básica de saúde; a importância de diminuir os fatores de risco que predispõem a mulher a evoluir um quadro carcinógeno bem como a prática periódica de exames preventivos.

Para Melo et al (2012) os profissionais de enfermagem, em especial aqueles que atuam na atenção básica onde muitas vezes se não em sua grande maioria o quadro de câncer de colo de útero é descoberto por meio de exame ginecológico Papanicolau, é de total relevância o conhecimento e a importância de se evitar essa patologia por meio da promoção da saúde no ato da consulta de enfermagem, sendo que este aconselhamento deve ser individualmente bem como em campanhas coletivas com o intuito de afastar as mulheres dos fatores de risco e incentivar a ida periódica ao sistema de saúde.

Segundo Fernandes, Janicas e Narchi (2007) o câncer cérvico-uterino há muitas décadas vem sendo alvo de atenção da comunidade científica por ocupar lugar de destaque nas elevadas taxas de morbidade e mortalidade entre a população feminina, especialmente nos países em desenvolvimento, nos quais esse tipo de câncer relaciona-se ao perfil epidemiológico das mulheres, à frequência de risco e, sobretudo, ao grau de implementação de ações efetivas de curto e longo prazos em todos os níveis de atenção.

Partindo dessa contextualização, a problemática desta pesquisa é analisar a importância da prevenção do câncer de colo de útero; qual o papel da enfermagem na promoção da prevenção dessa patologia e quais os métodos preventivos dos quais a mulher pode ser utilizar nessa prevenção e a importância da realização periódica de exames preventivos.

O presente artigo tem como objetivo geral avaliar os artigos publicados na base de dados SCIELLO, na área da enfermagem e revista especializadas em saúde da mulher, demonstrar como a enfermagem é importante na atenção básica com relação à prevenção do câncer de colo de útero na mulher. Tendo como objetivos específicos identificar as pesquisas relacionadas à prevenção do câncer do colo de útero; observar quais as contribuições apresentadas nas pesquisas em estudo e demonstrar a importância da detecção precoce na atenção básica na maioria das vezes feita pelo profissional da enfermagem.

Percebe-se uma grande preocupação nos temas sobre qualidade de vida das mulheres em virtude dos altos índices de morbimortalidade relacionado ao câncer cervico-uterino, neste sentido que esse artigo através de um rastreamento bibliográfico 
vem elucidar e contribuir tratando de um fenômeno que ocorre dentro da organização.

Este estudo justifica-se pela contribuição no entendimento da importância da prevenção do câncer do colo uterino e como a enfermagem na atenção básica tem um papel relevante na promoção da saúde. São vastos os estudos voltados a este tema oque demonstra sua relevância na área das pesquisas voltada a métodos de prevenção e promoção da saúde da mulher.

A relevância deste estudo abrange também em identificar artigos na área da enfermagem sobre a prevenção e detecção precoce do câncer do colo uterino; descrever e observar quais as contribuições destes estudos para o aperfeiçoamento das metodologias da enfermagem no rastreamento e diagnostico de casos precoces de câncer cérvico-uterino e promoção de saúde voltada a mulher em seu período de risco a fatores que predisponha a um possível surgimento do C.A uterino.

A importância desta pesquisa está diretamente relacionada a três aspectos fundamentais: promover a realização periódica de exames com fins de se evitar a doenças bem como um diagnostica tardio; fazer com que a enfermagem avaliar suas condutas mediante suas ações que são de total importância na atenção básica no que diz respeito à saúde da mulher e por fim construir novas metodologias de atuação no combate ao câncer cérvico-uterinos para que venha contribuir de forma qualitativa na vida social das mulheres.

\section{REFERENCIAL TEÓRICO}

A atuação da enfermagem dentro da promoção e prevenção do câncer cérvico da na atenção básica é de total importância, a partir do pressuposto que a formação que o enfermeiro possui em sua vida acadêmica dá ao mesmo uma visão multilateral do meio social, o que facilita sua atuação na socialização de qualquer membro da sociedade.

Visando promover e proteger a saúde da mulher, criou-se então o Programa "Assistência Integral à saúde da Mulher: bases de ação programática” (PAISM) o qual foi elaborado pelo Ministério da Saúde e apresentado na Comissão Parlamentar Mista de Inquérito (CPMI) da explosão demográfica em 1983, a discussão se pautava predominantemente sobre o controle da natalidade. O Ministério da Saúde teve um papel fundamental, pois influenciou no âmbito do Governo Federal e este por sua vez, se posicionou e defendeu o livre arbítrio das pessoas e das famílias brasileiras em relação a quando, quantos e qual o espaçamento entre os/as filhos/as.

O Instituto Nacional do Câncer (INCA), afirma que dentre tantas doenças que acometem a mulheres, o câncer é uma das mais graves; refere-se a um conjunto de mais 
de 100 doenças que têm em comum o crescimento desordenado de células, que invadem tecidos e órgãos. Dividindo-se rapidamente, estas células tendem a ser muito agressivas e incontroláveis, determinando a formação de tumores malignos, que podem espalhar-se para outras regiões do corpo. As causas de câncer são variadas, podendo ser externas ou internas ao organismo, estando inter-relacionadas. As causas externas referem-se ao meio ambiente e aos hábitos ou costumes próprios de uma sociedade. As causas internas são, na maioria das vezes, geneticamente pré-determinadas, e estão ligadas à capacidade do organismo de se defender das agressões externas.

De acordo com Melo et al (2012) no Brasil, o câncer do colo do útero é a terceira neoplasia maligna que acomete as mulheres, apenas superado pelos cânceres de pele não melanoma e da mama. Foram estimados cerca de dezoito mil novos casos para os anos de 2012 e 20131. Sua frequência é variável conforme a região e implica em que ocupe as seguintes posições no país: Norte - primeira; Centro-Oeste e Nordeste - segunda; Sudeste terceira e no Sul a quarta. Considerando-se somente a população feminina que está em segundo lugar e representa cerca de $15 \%$ de todos os tipos de câncer.

A Associação Brasileira de Portadores de Câncer (AMUCC) relata que prevenção do câncer nem sempre é possível, mas há fatores de risco que estão na origem de diferentes tipos de tumor.

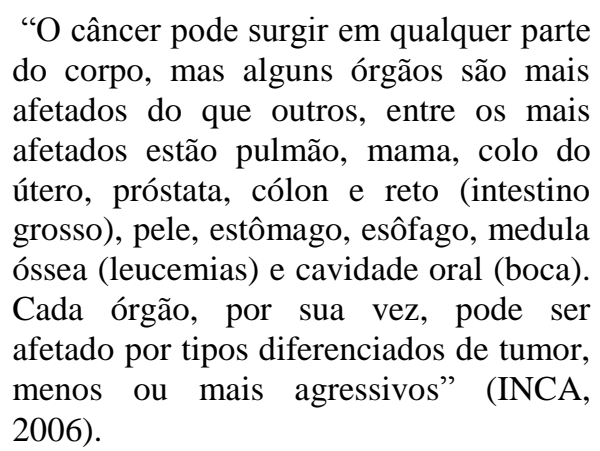

De acordo com Fernandes, Janicas e Narchi (2007, p. 127) o início do câncer cérvico-uterino tem início com uma lesão intra-epitelial progressiva que pode evoluir para um câncer invasivo em um prazo de 10 a 20 anos, caso não seja oferecido tratamento. Nesse período de evolução, a doença passa por fases pré-clínicas detectáveis e curáveis, o que lhe confere um dos mais altos potenciais de prevenção e de cura.

Segundo Macedo, Filho e Magalhães (2011) uma das principais alterações que podem levar a mulher a desenvolver um câncer cérvico-uterino é a infecção pelo papilomavírus humano, o HPV, com alguns subtipos de alto risco e relacionados a tumores malignos.

Instituto Nacional do Câncer (INCA) relata que o câncer do colo do útero, também chamado de cervical, demora muitos anos para se desenvolver. As alterações das células que podem desencadear o câncer são descobertas facilmente no exame preventivo (conhecido também como Papanicolau), por isso é importante a sua realização periódica. 
Melo (2012) aponta que a prevenção primária do câncer cérvico-uterino está diretamente ligada à redução da exposição aos fatores de riscos. O Programa de Saúde da Família (PSF) tem importante papel na identificação de grupos de mulheres com perfil de risco para desenvolver o câncer cérvico-uterino e, com base nas necessidades levantadas, programar ações de intervenção no meio ambiente e em seus fatores de risco. Enquanto que a prevenção secundária do câncer cérvico-uterino é realizada pelo exame citopatológico para detecção do câncer in situ ou das lesões precursoras.

\begin{abstract}
"O exame preventivo do câncer do colo do útero (PCCU), também conhecido como Papanicolau, é um exame simples que foi criado pelo Dr. George Papanicolau em 1940. O sucesso do teste é porque ele pode detectar doenças que ocorrem no colo do útero antes do desenvolvimento do câncer. $\mathrm{O}$ exame não é somente uma maneira de diagnosticar a doença, mas serve principalmente para determinar o risco de uma mulher vir a desenvolver o câncer" (ORQUIZA, 2013).
\end{abstract}

O INCA comenta que o PCCU é a principal estratégia para detectar lesões precursoras e fazer o diagnóstico da doença. O exame pode ser feito em postos ou unidades de saúde da rede pública que tenham profissionais capacitados. É fundamental que os serviços de saúde orientem sobre o que é e qual a importância do exame preventivo, pois sua realização periódica permite reduzir a mortalidade por câncer do colo do útero.
Merighi, Hamano e Cavalcante (2002, p. 2) reforçam que os profissionais devem ter consciência, no ato do exame, que cada pessoa tem sua própria percepção sobre os procedimentos que envolvem a prevenção do câncer cérvico-uterino. Um procedimento simples, rotineiro, rápido e indolor aos olhos do profissional, pode ser visto pela mulher como procedimento agressivo, físico e psicologicamente, pois a mulher que busca o serviço traz consigo suas bagagens social, cultural, familiar e religiosa.

Acredita-se que o presente trabalho, de alguma forma, vem contribuir para proporcionar reflexões e um maior entendimento acerca do combate, promoção e prevenção do câncer de útero, uma vez que o conhecimento dos fatores desencadeadores dessa patologia, bem como de suas conseqüências, podem fazer com que as mulheres se conscientizem da importância do autocuidado e da busca periódica pelo serviço de saúde.

\section{METODOLOGIA}

Trata-se de uma pesquisa bibliográfica de cunho qualitativo com a finalidade de analisar as pesquisas realizadas acerca do tema proposto. Onde foi utilizado como base de dados lonline SciELO (Scientific Electronic Library Online), que consiste em "uma biblioteca eletrônica que abrange uma coleção selecionada de periódicos científicos 
brasileiros", livros e sites do Ministério da Saúde.

De acordo com Lakatos e Marconi (2006), a pesquisa bibliográfica constitui-se em um levantamento, seleção e documentação de toda bibliografia já publicada sobre o assunto que está sendo pesquisados, em livros, revistas, jornais, boletins, monografias, teses, dissertações, material cartográfico, com o objetivo de colocar o pesquisador em contato direto com todo o material já descrito sobre a temática.

Para a elaboração desta revisão de literatura, foram consultados periódicos indexados ao SciELO (Scientific Eletronic Library Online), sites eletrônicos do Ministério da saúde e livros que tratem do tema proposto. Foram utilizados os seguintes descritores: câncer cérvico-uterino; prevenção do câncer de colo de útero; atuação da enfermagem no PSF. Para isto, incluiremos publicações acerca do tema no período de $2006-2013$.

Foram consideradas as publicações relacionadas a métodos de prevenção do desenvolvimento do câncer de colo de útero; atuação da enfermagem no combate ao câncer cérvico-uterino; a importância da promoção e prevenção do câncer de colo de útero por parte da enfermagem no PSF.

A escolha dos artigos foi realizada mediante uma leitura previa dos resumos, a fim de confirmar a temática proposta. Os artigos selecionados foram analisados e escolhidos de acordo com as seguintes categorias previamente definidas: métodos de prevenção do desenvolvimento do câncer de colo de útero; atuação da enfermagem no combate ao câncer cérvico-uterino; a importância da promoção e prevenção do câncer de colo de útero por parte da enfermagem no PSF.

Após o mapeamento dos dados, os artigos foram analisados e tabulados conforme os enfoques priorizados, e após tabulações de recortes de nosso interesse os mesmos tiveram seus aspectos descritos, analisados e expostos no corpo de construção da presente pesquisa bibliográfica.

\section{ANALISE E RESULTADOS}

Em varias pesquisas analisadas as Unidades de Atenção Primária à Saúde (UAP'S) são consideradas porta de entrada do usuário no sistema de saúde, espaço em que o enfermeiro é importante integrante da equipe multiprofissional da Estratégia Saúde da Família (ESF), aspecto este também intimamente relacionado a detecção de muitos casos de câncer de colo de útero ser detectado nas UAP'S pelo profissional da enfermagem durante o exame citológico de rotina.

De acordo com Brasil (2010) a equipe que compõem essas UAP'S é conforme o tamanho da área de abrangência se distribuem equipes que têm como desafio o trabalho integrado e a responsabilidade pelas pessoas ali residentes. Nesse contexto, os enfermeiros 
exercem atividades técnicas específicas de sua competência, administrativas e educativas e através do vínculo com as usuárias, concentra esforços para reduzir os tabus, mitos e preconceitos e buscar o convencimento da clientela feminina sobre os seus benefícios da prevenção.

Mediante o que foi citado por Brasil (2010) anteriormente o papel do profissional da enfermagem dentro dessa UAP'S é de total relevância em virtude de ser um profissional que está em contato direto e continuo com os pacientes, podendo assim detectar problemas por meios de sua consulta de enfermagem.

De acordo com o Instituto Nacional de Câncer (2008) para o planejamento das atividades e estratégias, são consideradas e respeitadas às peculiaridades regionais, envolvimento das lideranças comunitárias, profissionais da saúde, movimentos de mulheres e meios de comunicação.

A relevância das ações desenvolvidas pelo enfermeiro em sua atuação nas equipes da ES F no exercício da prevenção e a promoção da saúde se delineou o objeto deste estudo. A inquietação se direcionou ao cotidiano assistencial deste profissional quanto à realização do exame de Papanicolaou como estratégia de redução dos danos, a partir da detecção precoce da doença e consequente melhoria da qualidade de vida das mulheres.

Este olhar que considera as atribuições do enfermeiro teve como objeto de pesquisa o agir desse profissional no cenário da atenção primária à saúde no contexto das estratégias preventivas, com o objetivo de analisar o desenvolvimento da prevenção e detecção precoce do câncer do colo do útero no cotidiano assistencial da enfermeira que atua nas equipes da ESF, a partir de suas atribuições, propostas pelo Ministério da Saúde.

\section{CONSIDERAÇÕES FINAIS}

Conclui-se que o conhecimento cientifico dos profissionais na enfermagem dentro da ESF é de total relevância e importância, pois quando esse profissional detêm conhecimento cientifico relacionado aos possíveis achado que suas atitudes preventivas pode se deparar, este profissional consegue ter uma visão holística dos pacientes e assim desenvolver atividades preventiva, educativas e detectivas e em especial no que diz respeito câncer de colo de útero que é um patologia que até os dias atuais matam tantas mulheres.

\section{REFERÊNCIAS}

ARCHI, N. Z; JANICAS, R.C.S.V; FERNANDES, R. A.Q. Enfermagem e saúde da mulher. Prevenção e controle do câncer cérvico-uterino. São Paulo: Manole, 2007, p. 127.

Associação Brasileira de Portadores de Câncer. Prevenção do câncer. Disponível em: http://www.amucc.com.br/conteudo/prevenca o-do-cancer/. Acesso em 18 de Fev. 2013. 
BRASIL. Ministério da Saúde. Especial: Saúde garante mais proteção à saúde das mulheres. Disponível em: http://portal.saude.gov.br/portal/aplicacoes/ reportagensEspeciais/default.cfm?pg=dspDeta lhes\&id_area $=124 \& C O \_N O T I C I A=10007$. Acesso em 14 de Jun. 2013.

BRASIL. Instituto Nacional do Câncer. Câncer. Disponível em: http://www2.inca.gov.br/wps/wcm/connect/ca ncer/site/oquee/. Acesso em 14 .de Jun 2013.

Brasil. Ministério da Saúde. Secretaria de Atenção à Saúde. Departamento de Atenção Básica. Rastreamento. Brasília: Ministério da Saúde; 2010. 95 p. (Série A. Normas e manuais técnicos. Cadernos de Atenção Primária; n. 29).

LAKATOS, E M, MARCONI, M. A Metodologia Científica. 4 ed São Paulo: Atlas, 2006.

MELO, M.C.S.C; VILELA, F.; SALIMENA, A. M.O; SOUZA, I.E.O. O Enfermeiro na Prevenção do Câncer do Colo do Útero: o Cotidiano da Atenção Primária. Revista Brasileira de Cancerologia 2012; 58(3): 389398

MACEDO, M.H.H.A; FILHO, A.L.S; MAGALHÃES, I.M.Q.S. Prevenção de câncer de colo uterino: desafios de uma década. Com. Ciências Saúde - 22 Sup 1:S121-S128, 2011

ORQUIZA, Sônia. Orientações médicas. O que é exame de papanicolau. Disponível em http://www.orientacoesmedicas.com.br/defaul t.asp. Acesso em 20 de Fev. 2013.

Instituto Nacional de Câncer (Brasil). Nomenclatura brasileira para laudos cervicais e condutas preconizadas: recomendações para profissionais de saúde. Rio de Janeiro: INCA; 2006. 56 p.

Instituto Nacional de Câncer (Brasil). Ações de enfermagem para o controle do câncer: uma proposta de integração ensino-serviço. 3a ed. rev., atual. e ampl. Rio de Janeiro: INCA; $2008.628 \mathrm{p}$. 